\title{
Conhecimento das gestantes acerca das medidas de alívio da dor durante o parto
}

\author{
Knowledge of pregnant women about measures of pain \\ relief during childbirth
}

\author{
Alessa Sousa da Silva' • Carla Cristina Chaves de Oliveira ${ }^{2}$ Emmanuel Calisto da Costa Brito ${ }^{3}$ \\ Tiago Barreto Castro e Silva ${ }^{4}$ Danielle Rosa Evangelista ${ }^{5}$
}

\begin{abstract}
RESUMO
Objetivou-se analisar o conhecimento das gestantes primíparas acerca de métodos de alivio da dor no primeiro estágio do parto.Trata-se de estudo exploratório e transversal. Foram entrevistadas 49 gestantes cadastradas na rede municipal de Palmas, Tocantins. Os dados foram processados no programa estatístico Epi Info, versão 3.3 de 2004. A análise foi através de estatística descritiva simples. Os resultados mostram gestantes com 25,04 $\pm 5,7 \mathrm{I}$ anos de idade, com parceiro fixo, ensino médio completo, renda média de 1473, I4 4936 , I 8 e 7,02 $\pm 2,03$ consultas pré-natal, encontravam-se com 34,8 $\pm 3,42$ semanas de gestação. $100 \%$ afirmaram que o tema não foi abordado no acompanhamento pré-natal. $75,5 \%$ das gestantes foram classificadas com nenhum conhecimento. Concluiu-se que as gestantes apresentam necessidades de ter conhecimento sobre os métodos de alivio a dor, para o exercício do processo de parir de forma humanizada.

Palavras-chave: Primeira fase do Trabalho de Parto; Dor do Parto; Conhecimento.
\end{abstract}

\begin{abstract}
The objective of this study was to analyze the knowledge of primiparous pregnant women about methods of pain relief in the first stage of labor.This is an exploratory and cross-sectional study. Forty-nine pregnant women registered in the municipal network of Palmas, Tocantins were interviewed. The data was processed in the statistical program Epi Info, version 3.3 of 2004. The analysis was through simple descriptive statistics. The results show pregnant women who were $25.04 \pm 5.7 \mathrm{I}$ years old, with a fixed partner, complete high school, average income of $1473.14 \pm 936.18$ and $7.02 \pm 2.03$ prenatal consultations, were with $34.8 \pm 3.42$ weeks of gestation. I I $0 \%$ stated that the topic was not addressed in prenatal care. $75.5 \%$ of the pregnant women were classified with having no knowledge. It was concluded that the pregnant women present the need to be aware of the methods of pain relief, to exercise the process of giving birth in a humanized way.

Keywords: Labor Stage, First; Labor Pain; Knowledge.
\end{abstract}

'Acadêmica de Enfermagem da Universidade Federal do Tocantins (UFT). E-mail: alessasousasil@gmail.com;

Enfermeira. Mestre em Ciências da Saúde. Secretaria Estadual de Saúde do Tocantins. E-mail: carla_cristina_c@hotmail.com;

${ }^{3}$ Enfermeiro. Especialista em Saúde pública, Secretaria Municipal de Saúde de Palmas. E-mail: emmanuelcalistocb@gmail.com;

${ }^{4}$ Enfermeiro. Doutor em Enfermagem. Universidade Federal do Tocantins (UFT). E-mail: tiagobcs@uft.edu.br;

${ }^{5}$ Enfermeira. Doutora em Enfermagem. Universidade Federal do Tocantins (UFT). E-mail: daniellerosa@uft.edu.br. 


\section{INTRODUÇÃO}

As parturientes, principalmente as primíparas, geralmente não apresentam conhecimento sobre os eventos fisiológicos que ocorrem durante o trabalho de parto, com destaque para a dor causada pelas contrações uterinas e, consequentemente, não conhecem as medidas de alívio da dor neste período, o que pode tornar este momento único em uma experiência negativa para a mulher ${ }^{(1)}$.

É imprescindível que as gestantes e seus acompanhantes - sejam eles os (as) companheiros (as), membros da família ou seus amigos - recebam informações adequadas sobre as medidas de alívio da dor para minimizar os eventos fisiológicos no momento do parto. Estas informações devem ser transmitidas através de atividades de educação em saúde, pois muitas vezes este é o espaço em que se compartilham dúvidas e experiências que normalmente não são discutidas em consultas formais, dentro dos consultórios dos profissionais de saúde. Assuntos esses que devem abranger as orientações e incentivo para o parto normal, resgatando-se a gestação, o parto, - puerpério e o aleitamento materno como processos fisiológicos e sinais e sintomas do parto(l).

Sobre isto, estudo(2) aponta a necessidade de ações educativas de preparação para o parto, voltadas à humanização da assistência de forma a promover experiências positivas em relação ao parto por meio da construção do conhecimento crítico, incentivando a autonomia da mulher durante o parto e exercício da cidadania.

Apesar de o acesso das parturientes aos recursos não farmacológicos para o alívio da dor no trabalho de parto ser recomendado, a utilização destes na assistência obstétrica ainda não é rotina na grande maioria dos serviços, possivelmente pelo desconhecimento destes recursos e de seus possíveis benefícios, tanto pelos profissionais de saúde como pela população. A utilização desses recursos no trabalho de parto busca resgatar o caráter fisiológico da parturição ${ }^{(3)}$. O pré-natal apresenta-se como momento adequado para essas ações educativas em saúde e espera-se que a gestantes tenham conhecimento sobre todos os eventos fisiológicos do parto e sobre métodos ou medidas de alivio da dor ${ }^{(1)}$.

O parto é um evento fisiológico com início caracterizado pelas contrações uterinas e que se finda na primeira hora após a saída da placenta. Didaticamente, ele é dividido em quatro estágios/períodos. O primeiro período é o intervalo desde o início das contrações uterinas dolorosas até a dilatação completa. $O$ segundo período, compreende desde a dilatação completa até o desprendimento do feto. $O$ terceiro é o período de tempo entre o nascimento do feto e a expulsão da placenta. $O$ quarto período, é a primeira hora após a expulsão da placenta ${ }^{(4-5)}$.
Dentre estes estágios, o primeiro é o mais crítico, uma vez que as dores são mais intensas e frequentes. Dentre os eventos fisiológicos do primeiro estágio, destaca-se a dilatação e apagamento do colo, as contrações uterinas e a descida da apresentação fetal( ${ }^{(6)}$.

No primeiro estágio, a aplicação das medidas de alívio da dor é recomendada. Para este fim, são preconizados os seguintes métodos: adotar posturas e posições variadas, deambulação, respiração ritmada e ofegante, comandos verbais e relaxantes (estes auxiliam no desvio da atenção a dor), banho morno de chuveiro e/ou imersão, massagens e toques, uso de bola de parto para minimizar as dores ${ }^{(3-7)}$. Essas informações facilitam $\circ$ trabalho de parto, tanto para a parturiente como para os profissionais envolvidos, uma vez que ter o conhecimento dessas ações facilita o desenvolver desse processo.

O enfermeiro deve ter conhecimento adequado para estar orientando as gestantes durante as consultas de pré-natal a cerca dessas informações. $O$ conhecimento adquirido pelo profissional de enfermagem durante seu processo de formação acadêmica o possibilita orientar sobre o início do parto e seus estágios, visando principalmente o primeiro, pois se trata do período do parto propriamente dito onde ocorrem às dores mais intensas e todos os demais eventos fisiológicos para o desenvolvimento do parto, bem como a orientação sobre os métodos de alivio para a dor. Desta forma, a falta de informação das gestantes sobre os eventos fisiológicos que envolvem o parto e os métodos de alivio da dor auxiliam para uma experiência negativa deste momento. Com as orientações prestadas no período pré-natal, esses conhecimentos adquiridos podem tornar o parto uma experiência positiva e única na vida desta mulher ${ }^{(8)}$.

Diante do exposto, ou seja, que o primeiro estágio do parto é o mais crítico, são precisas intervenções, como a aplicação das medidas não farmacológicas para alívio da dor, medidas que são baseadas em evidências científicas, e é também necessário informar as mulheres sobre estas medidas, o que se justifica esse estudo.

A questão norteadora do estudo foi: Qual o nível de conhecimento que as gestantes têm sobre as medidas de alivio a dor no primeiro período do parto? Responder essa questão irá representar um progresso no conhecimento, pois culminará em evidência para a reorientação da atenção pré-natal, no sentido de identificar lacunas do conhecimento dessas gestantes que precisam de maior ênfase para os aspectos relacionados ao parto, para que possam apresentam nível de conhecimento satisfatório sobre as questões que envolvem o primeiro estágio do trabalho de parto, sendo este aspecto a relevância social da pesquisa.

O presente estudo teve como objetivo verificar o nível de conhecimento sobre os métodos de alivio para a dor. 


\section{MÉTODOS}

Trata-se de estudo exploratório e transversal, realizado município de Palmas-TO, em cinco Centros de Saúde da Comunidade (CSC), escolhidos por conveniência por realizarem atendimento pré-natal, configurando locais adequados para a realização deste estudo.

A coleta de dados ocorreu entre os meses de janeiro a outubro de 2015 , período pré-estabelecido, respeitando o prazo que a pesquisa tinha para ser concluída, por se tratar de um trabalho de conclusão do curso de graduação em enfermagem.A responsável pela coleta de dados realizou o levantamento da agenda do acompanhamento pré-natal, e nos dias destinados a essas consultas, compareceu até o local para realizar a coleta de dados. Todas as gestantes encontradas nos CSC nesse período, nos dias estabelecidos para coleta de dados, foram abordadas e convidadas a participar do estudo.

As gestantes que aceitassem participar eram avaliadas quanto o atendimento do critérios de seleção da amostra. Os critérios foram: ter idade maior ou igual a dezoito anos; ser primigesta; apresentar Idade Gestacional (IG) igual ou acima de 30 semanas no momento da coleta de dados; ter realizado no mínimo três consultas de acompanhamento pré-natal.Após aplicação do critérios de seleção, totalizaram 49 gestantes elegíveis à pesquisa.

A coleta de dados foi realizada por meio de entrevista, utilizando um formulário semiestruturado, elaborado pela equipe, que norteou a coleta de dados. $O$ instrumento versava sobre questões referentes à caracterização da amostra (idade, condição de união, escolaridade, renda e ocupação); caracterização obstétrica (número de consultas e IG); conhecimento sobre as medidas de alivio da dor; abordagem no pré-natal sobre essas medidas; e se a gestante considera-se esclarecida sobre $o$ assunto.

Destaca-se que a entrevista para coleta de dados só era iniciada após leitura e assinatura do Termo de Consentimento Livre e Esclarecido pela gestante.

O ambiente no qual foram coletados os dados foi calmo e reservado, de forma a manter a privacidade, na própria sala de espera do CSC.A coleta de dados não afetou a rotina do CSC, visto que foi realizada no momento em que a gestante aguarda $\circ$ atendimento, não comprometendo a assistência prestada ao usuário.

Os dados foram processados no programa estatístico Epi Info, versão 3.3 de 2004. A análise foi feita através de estatística descritiva simples, utilizando frequência absoluta e relativa, média, desvio padrão.

Para avaliar o nível de conhecimento das mulheres, foi utilizada uma escala tipo likert adaptada, com fundamentação teórica, à escala adotada em estudo que objetivou identificar o nível de conhecimento sobre os cuidados pré-concepcionais e riscos maternos e fetais em mulheres portadoras de diabetes mellitus ${ }^{(9)}$. Tal escala foi esco-
Ihida por se tratar de um instrumento da Enfermagem e já ter sido validada e aceita no meio acadêmico, o que confere credibilidade à escala.

A escala é composta por cinco itens para avaliação do conhecimento acerca do métodos de alivio a dor: I- NENHUM; 2- LIMITADO; 3- MODERADO; 4- SUBSTANCIAL; 5- EXTENSO. Sendo estes adaptados à temática da pesquisa em questão, criando-se um sistema de pontuação de cada item da escala.

Para esta escala sobre métodos de alivio da dor, foram adotados oito critérios, mediante levantamento da literatura, a saber: Adotar posturas, posições variadas; Deambulação; Respiração ritmada e ofegante; Comandos verbais e relaxantes; Banho morno de chuveiro e imersão; Massagens e toques; Uso de bola de parto;Alimentação líquida livre durante o parto.

Quando a mulher não citou nenhum método de alivio da dor, recebeu a pontuação I da escala, ou seja, nenhum conhecimento. Foi considerada com conhecimento limitado, recebendo a pontuação 2 da escala, a mulher que citou entre um e três métodos de alivio. Foi considerada com conhecimento moderado, recebendo a pontuação 3 da escala, a mulher que citou quatro métodos de alivio. Foi considerado com nível substancial de conhecimento, a mulher que citou de cinco a sete métodos de alivio, recebendo a pontuação 4 da escala e foi considerado extenso, ou seja, a pontuação 5 da escala, quando a mulher citou os 8 critérios de avaliação do conhecimento dos métodos de alivio a dor estabelecidos.

O projeto foi submetido ao Comitê de Ética em Pesquisa da Universidade Federal do Tocantins sob o parecer favorável 069/2014.

\section{RESULTADOS}

A caracterização da amostra é apresentada na tabela $I$.

A ocupação das mulheres mostrou que 18 (36,7\%) trabalhavam no setor privado e $15(30,6 \%)$ afirmaram ser do lar, sendo estas as ocupações mais frequentes. As outras ocupações que surgiram na coleta de dados foram, para 9 (18,4\%) servidoras públicas e 7 (14,3\%) que afirmaram ser estudantes.

A caracterização obstétrica da amostra estudada é apresentada na tabela 2 .

O nível de conhecimento sobre as medidas não farmacológicas de alívio de dor estão descritas na tabela 3.

A distribuição do número de gestantes de acordo a caracterização da fonte de conhecimento, abordagem no pré-natal e percepção, em CSC de Palmas -TO, neste estudo mostrou que 49 (100\%) das gestantes afirmaram que o assunto objeto deste estudo não foi abordado no acompanhamento pré-natal. Quando questionadas em relação a consideração que faziam sobre sentir-se esclarecida sobre a temática 49 (100\%) das gestantes afirmaram que não se sentiam esclarecidas. 
TABELA 1 - Distribuição do número de gestantes de acordo com as características sóciodemográficas em CSC de Palmas -TO, Brasil, 2015.

\begin{tabular}{l|c|c}
\hline Variáveis & $\mathbf{n}$ & $\%$ \\
\hline Idade $(\bar{x}=25,04 ; S=5,71)$ & & 42,9 \\
18 a 22 & 11 & 22,4 \\
23 a 27 & 13 & 26,5 \\
28 a 33 & 4 & 8,1 \\
34 a 38 & & \\
\hline Condição de União & 36 & 73,5 \\
\hline Parceiro Fixo & 13 & 26,5 \\
Parceiro Eventual & & \\
\hline Escolaridade & 1 & 2,0 \\
\hline Fund. Incompleto & 3 & 6,1 \\
Fund. Completo & 12 & 24,5 \\
Méd. Incompleto & 19 & 38,8 \\
Méd. Completo & 8 & 16,3 \\
Sup. Incompleto & 6 & 12,2 \\
Sup. Completo & & \\
\hline Renda $(\bar{x}=1473,14 ; S=936,18)$ & 28 & 57,1 \\
\hline 724 a 1300 & 11 & 22,3 \\
1400 a 2100 & 10 & 20,2 \\
2300 a 5000 & & \\
\hline
\end{tabular}

TABELA 2 - Distribuição do número de gestantes de acordo com número de consultas e idade gestacional (IG) em CSC de Palmas -TO, Brasil, 2015.

\begin{tabular}{l|c|c}
\hline Variáveis & $\mathbf{n}$ & $\%$ \\
\hline Número de Consultas $(\bar{x}=7,02 ; S=2,03)$ & & \\
\hline$<6$ & 11 & 22,4 \\
6 & 12 & 24,5 \\
$>6$ & 26 & 53,0 \\
\hline IG $(\bar{x}=34,81 ; S=3,42)$ & & \\
\hline 30 a 33 & 20 & 40,9 \\
34 a 37 & 16 & 32,6 \\
38 a 40 & 13 & 27,4 \\
\hline
\end{tabular}

\section{DISCUSSÃo}

Com relação à idade das gestantes encontrada nesse estudo esperava-se uma idade mínima de 18 anos, pois esse era um dos critérios de inclusão da pesquisa. A média de idade encontrada neste estudo equipara-se a de outros estudos sobre medidas de alívio da dor em gestantes ${ }^{(10-11)}$. Percebe-se que a maioria estudada encontrava-se em idade recomendada para engravidar, diminuindo riscos à saúde materno-fetal ${ }^{(12)}$. Pesquisas envolvendo a caracterização do atendimento pré-natal revelam que a maior idade das gestantes está associada à maior adesão ao pré-natal e um maior conhecimento sobre sua impotência ${ }^{(13)}$.
A estabilidade conjugal foi encontrada na maioria das gestantes entrevistadas. Sobre isso, a estabilidade conjugal é descrita na literatura como uma influência positiva, pois o acompanhamento do companheiro tem fundamental importância para o sucesso do pré-natal e do parto(14).

Quanto à escolaridade, as gestantes eram alfabetizadas. O nível de instrução deve ser analisado durante a consulta pré-natal, pois pode influenciar na compreensão das informações fornecidas durante a consulta, inclusive sobre os hábitos de vida saudável, refletindo no cuidado com a família e com a gestação(12).

Em relação à renda familiar, pode-se afirmar que as mulheres possuem baixo poder econômico, visto que 
TABELA 3 - Distribuição do número de gestantes de acordo com o nível de conhecimento sobre as medidas de alivio a dor, em CSC de Palmas -TO, Brasil, 2015.

\begin{tabular}{|c|c|c|}
\hline Variáveis & $\mathrm{n}$ & $\%$ \\
\hline \multicolumn{3}{|c|}{ Conhece algum método de alivio para as dores do parto } \\
\hline Sim & 12 & 24,5 \\
\hline Não & 37 & 75,5 \\
\hline \multicolumn{3}{|c|}{ Conhecimento dos métodos } \\
\hline \multicolumn{3}{|c|}{ Posição Variada } \\
\hline Sim & 3 & 6,1 \\
\hline Não & 46 & 93,9 \\
\hline \multicolumn{3}{|c|}{ Deambulação } \\
\hline Sim & 5 & 10,2 \\
\hline Não & 44 & 89,8 \\
\hline \multicolumn{3}{|l|}{ Respiração } \\
\hline Sim & 5 & 10,2 \\
\hline Não & 44 & 89,8 \\
\hline \multicolumn{3}{|c|}{ Comandos verbais e relaxantes } \\
\hline Sim & 0 & 0 \\
\hline Não & 49 & 100,0 \\
\hline \multicolumn{3}{|c|}{ Banho morno } \\
\hline Sim & 5 & 10,2 \\
\hline Não & 44 & 89,8 \\
\hline \multicolumn{3}{|c|}{ Massagem e toque } \\
\hline Sim & 1 & 2,0 \\
\hline Não & 48 & 98,0 \\
\hline \multicolumn{3}{|c|}{ Bola de parto } \\
\hline Sim & 0 & 0 \\
\hline Não & 49 & 100,0 \\
\hline \multicolumn{3}{|c|}{ Alimentação liquida } \\
\hline Sim & 0 & 0 \\
\hline Não & 49 & 100,0 \\
\hline Escala & $\mathrm{n}$ & $\%$ \\
\hline Nenhum & 37 & 75,5 \\
\hline Limitado & 12 & 22,5 \\
\hline Moderado & 1 & 2,0 \\
\hline Substancial & 0 & 0 \\
\hline Extenso & 0 & 0 \\
\hline
\end{tabular}

a maioria relatou renda de até um pouco superior a um salário mínimo e algumas relataram que não tinham nenhum tipo de renda, o que é considerado um fator de risco para o aparecimento de complicações durante a gravidez e de terem filhos com baixo peso ou prematuros ${ }^{(12)}$.

Em relação à ocupação, a maioria das gestantes afirmou dedicar-se a cuidar do lar, refletindo na diminuição da renda familiar, corroborando com os achados de estudo realizado em Fortaleza- Ceará ${ }^{(12)}$. Em contrapartida, essa característica favorece o aleitamento materno, pois a inserção da mulher no mercado de trabalho é considerada um dos motivos para o desmame precoce, tendo em vista que a licença-maternidade, geralmente, é de quatro meses, e as técnicas de ordenha e armazena- mento do leite materno não são largamente divulgadas e ensinadas às mães ${ }^{(12)}$.

Estudo realizado com puérperas mostra que a adesão ao pré-natal é maior quanto maior o nível de escolaridade, é essa maior adesão tem relação direta como a renda familiar e a ocupação das gestantes ${ }^{(15)}$.

A caracterização das mulheres deste estudo, em relação a idade, escolaridade, e estado conjugal mostrou predomínio de gestantes jovens, com companheiro e escolaridade de baixa a média.

Em relação às consultas de pré-natal, o número de seis consultas pré-natais foi tomado como parâmetro, pois é o valor mínimo de consultas que o Ministério da Saúde recomenda como requisito mínimo de qualidade, corroborando com os achados de outros estudos ${ }^{(16-18)}$. 
De acordo com o Ministério da Saúde, toda mulher tem direito a ser orientada sobre as modificações e acontecimentos a ser dar no período gravídico-puerperal e o dever dessa orientação cabe aos profissionais da saúde envolvidos na assistência nesse período( ${ }^{(1)}$. Deveriam ser realizadas precocemente, consistir de encontros terapêuticos, educativos e interdisciplinares para maior compreensão das vivências, expressão de sentimentos e dúvidas, avaliação do bem-estar materno-fetal, preparação para o parto, a maternidade e paternidade; envolver as relações familiares, conjugais, os diálogos entre gestantes e com os profissionais. Essas ações realizadas durante o período de pré-natal das gestantes deixariam-nas mais bem informadas sobre cada momento de sua gestação, quando ao seu parto e o período pós-parto, tornando-as mais proativas e menos inseguras nesses períodos.

O conhecimento sobre as medidas não farmacológicas para alívio da dor no parto foi limitado. Existe evidência que esses são métodos eficazes para aliviar a dor no trabalho de parto, pois além de diminuírem a percepção dolorosa, ainda reduzem os níveis de ansiedade e estresse da mulher ${ }^{(19)}$.

A informação sobre métodos não farmacológicos de alívio da dor durante o trabalho de parto reforça a preocupação dos profissionais de saúde envolvidos na assistência em oferecer conforto e apoio às parturientes no enfrentamento da dor, como garantia de direito reprodutivo desta mulher. A satisfação da mulher com o seu parto não está relacionada somente à ausência da dor, mas às condições oferecidas para o seu enfrentamento. Os efeitos do suporte à mulher estão associados à duração do trabalho de parto, à redução de cesarianas e de partos vaginais instrumentais, à utilização de analgesia intraparto e melhores índices de Apgar no quinto minuto(20).

Estudo realizado com o objetivo de avaliar a relação entre assistência pré-natal e orientações para - parto na Atenção Primária a Saúde evidenciaram que entre as mulheres que realizaram mais de seis consultas, $81,7 \%$ relataram não ter recebido orientações para o parto durante o pré-natal $\left.\right|^{(18)}$. Destaca-se que os dados encontrados foram superiores, uma vez que $100 \%$ das gestantes afirmaram não ter recebido orientação no pré-natal, refletindo assim na falta de segurança em sentir-se esclarecidas sobre as medidas de alívio da dor no parto. Apesar das entrevistadas encontrarem-se ainda gestantes, a idade gestacional média foi $34,8 \pm 3,42$ semanas, ou seja, em período gestacional avançado, logo, deveriam ter recebido informações sobre o momento do parto.

O fato de $100 \%$ das mulheres não receberem nenhuma orientação a respeito das medidas não farmacológicas de alívio da dor no parto durante o pré-na- tal, remete a manutenção de um paradigma assistencial centrado na patologia e na queixa da usuária, em detrimento de um pré-natal com troca de informações referentes às questões da gravidez, parto, do puerpério e dos cuidados à criança ${ }^{(16)}$.

Diante das principais transformações fisiológicas durante a gestação bem como sua resolução fisiológica através do parto, os profissionais das equipes de saúde devem estar capacitados para prestar uma adequada assistência de pré-natal em todos os níveis de atenção à saúde da mulher, de modo a identificar as lacunas de conhecimento das mulheres e o seu adequado manejo, esclarecendo, de forma individual e/ou coletiva, suas necessidades. É importante, para as unidades de saúde, adequar o número de atendimentos no prénatal e grupos educativos, a fim de possibilitar maior tempo para esclarecimentos sobre gestação, parto e nascimento.

\section{CONCLUSÃO}

Essa pesquisa evidenciou que lacunas no nível de conhecimento das gestantes sobre as medidas de alivio a dor durante o parto. Faz-se necessário que 100\% das gestantes tenham conhecimento adequado e satisfatório sobre os métodos de alivio a dor, pois uma vez que elas dominarem sobre essa temática, seus benefícios, modos e momentos certos de se estar utilizando deles elas se tornam mais confiantes para o momento do parto e mais proativa no nesse momento de extrema importância em sua vida.

Os profissionais da saúde precisam se atentar quanto a essa falta de conhecimento, necessidade de ser feita essa orientação e elaborar estratégias para que esse conhecimento seja aderido e posto em prática por todas as mulheres.

Foram limitações desse estudo o quantitativo de muIheres encontradas nas unidades. Portanto, ressalta-se aqui a importância de realizar novos estudos com um número maior de gestantes, analisando a necessidade de incluir nestes estudos estratégias educativas com o objetivo de elevar o nível de conhecimento destas, promovendo a prática dos métodos de alivio a dor, consequentemente tornando o parto um momento mais prazeroso, como diminuição da percepção dolorosa e de menor ansiedade e estresse.

Os resultados evidenciaram a necessidade de ações voltadas para a saúde da mulher, no que diz respeito ao pré-natal abrangendo métodos não farmacológicos para alívio da dor, entre outros assuntos pertinentes ao ciclo gravídico puerperal. Evitando assim, possíveis experiências negativas com o parto que terão impactos não somente na vida das mulheres mas também na assistência ofertada e na saúde pública. 


\section{REFERÊNCIAS}

I.Secretaria de Atenção à Saúde, Ministério da Saúde. Atenção ao Pré-Natal de Baixo Risco. Brasília (Brasil): Ministério da Saúde, 2012. 316 p.

2.Silva LM, Barbieri M, Fustinoni SM.Vivenciando a experiência da parturição em um modelo assistencial humanizado. Rev Bras Enferm 201 I;64(I Suppl I): S 60-5.

3.Gallo RBS, Santana LS, Marcolin AC, Ferreira CHJ, Duarte G, Quintana SM. Recursos não-farmacológicos no trabalho de parto: protocolo assistencial. Femina 201 I;39(I):4I-8.

4.Lowdermilk DL. Processos do trabalho de Parto e Parto. In: Lowdermilk DL, Perry SE, Cashion K, Alden KR, editors. Saúde da Mulher e Enfermagem Obstétrica. Rio de Janeiro: Elsevier; 2012. p.365-8I.

5.Galão SH, Salazar CC, Freitas F. Mecanismo do Parto. In: Freitas F, Martins-Costa SH, Ramos JGL, Magalhães JA, editors. Rotinas em Obstetrícia. Porto Alegre:Artmed; 20I I. p. 30I-9.

6.Montenegro CAB, Rezende Filho J, editors. Rezende, Obstetrícia Fundamental. Rio de Janeiro: Guanabara Koogan; 201 I.

7.Organização Mundial da Saúde (OMS). Guia de prática clínica sobre cuidados com parto normal. 2010.

8.Quadros JS, Reis TLR, Colomé JS. Enfermagem obstétrica e educação em saúde: contribuições para vivência do processo de parturição. Rev Rene 2016;17(4): 45।-8.

9.Moura ERF, Evangelista DR, Damasceno AKC. Conhecimento de mulheres com diabetes mellitus sobre cuidados préconcepcionais e riscos materno-fetais. Rev Esc Enferm USP 2012;46(I): 22-9.

10. Mamede FV, Almeida AM, Souza I, Mamede MV. Pain during the labor active phase: the effect of walking. Rev Latino-am Enfermagem 2007;15(6): II57-62.

I I. Tostes NA, Seidl EMF. Expectativas de Gestantes sobre o Parto e suas Percepções acerca da Preparação para o Parto. Temas psicol 2016;24(2): 68I-93.
12. Peixoto CR, Lima TM, Costa CC, Freitas LV, Oliveira AS, Damasceno AKC. Perfil das gestantes atendidas no serviço de pré-natal das unidades básicas de saúde de Fortaleza-Ce. Rev Min Enferm 2012;16(2): I7I-I77.

13. Costa CSC, Vila VSC, Rodrigues FM, Martins CA, Pinho LMO. Características do atendimento pré-natal na rede básica de saúde. Rev Eletr Enf 2013:15(2): 516-22.

14. Oliva TA, Nascimento ER, Santo FRE Percepções e experiências de homens relativas ao pré-natal e parto de suas parceiras. Rev Enferm UERJ 2010;18(3): 435-40.

15. Líbera BD, Saunders C, Santos MMAS, Rimes KA, Brito FRSS, Baião MR. Avaliação da assistência pré-natal na percepção de puérperas e profissionais de Saúde. Ciênc saúde soletiva 201 I; I6(2): 4855-64.

16. Rodrigues AP, Padoin SMM, Aldrigui JD, Paula CC, Ximenes LB. Caracterização sóciodemográfica e obstétrica de puérperas internadas em alojamento conjunto no Brasil. Cienc enferm 2016;22(I): II3-123.

17. Santos LV, Lara MO, Lima RCR, Rocha AF, Rocha EM, Glória JCR et al. História gestacional e características da assistência pré-natal de puérperas adolescentes e adultas em uma maternidade do interior de Minas Gerais, Brasil. Ciênc saúde coletiva 2018;23(2): 6I7-25.

18. Gonçalves MF, Teixeira BEM, Silva MAS, Corsi NM, Ferrari RAP, Pelloso SM et al. Pré-natal: preparo para o parto na atenção primária à saúde no sul do Brasil. Rev Gaúcha Enferm 2017;38(3): 2016-0063.

19. Osório SMB, Santos Júnior LG, Nicolau AIO Avaliação da efetividade de métodos não farmacológicos no alivio a dor do parto. Rev Rene 2014;I5(I): I74-84.

20. Progianti JM, Mouta RJO.A enfermeira obstétrica: agente estratégica na implantação de práticas do modelo humanizada em maternidades. Rev enferm UERJ 2009: I7(2): 165-9. 